\title{
Sleep-Related Eating Disorder in Two Patients with Early-Onset Parkinson's Disease
}

\author{
Manoel Alves Sobreira Neto ${ }^{a}$ Márcio Alexandre Pena Pereira ${ }^{a}$ \\ Emmanuelle Silva Tavares Sobreira ${ }^{a}$ Marcos Hortes Nishiara Chagas ${ }^{a}$ \\ Guilherme Riccioppo Rodrigues $^{\mathrm{a}}$ Regina Maria França Fernandes ${ }^{\mathrm{a}}$ Vítor Tumas $^{\mathrm{a}}$ \\ Carlos H. Schenck ${ }^{\mathrm{b}}$ Alan Luiz Eckeli ${ }^{\mathrm{a}}$ \\ a Department of Neuroscience and Behavior, Ribeirão Preto School of Medicine, University of São Paulo, São Paulo, \\ Brazil; binnesota Regional Sleep Disorders Center, Department of Psychiatry, Hennepin County Medical Center \\ and University of Minnesota Medical School, Minneapolis, Minn., USA
}

Dear Sir,

Nocturnal eating disorders involve nearly nightly feeding episodes during either multiple nocturnal awakenings or during partial arousals from sleep, and are defined as excessive caloric intake with loss of control over consumption [1]. There are two major subtypes, one viewed more as an insomnia disorder, and the other more as a parasomnia. Nocturnal eating syndrome (NES) is characterized by the consumption of $50 \%$ or more of daily calories after the evening meal and/or eating after awakenings from sleep, with morning anorexia [1]. NES is considered a circadian delay of food intake with normal circadian timing of sleep onset [2]. Unrestrained binge eating is uncommon with NES, which is viewed as an insomnia disorder, with multiple, modest feedings being considered to occur to help re-induce sleep. Sleep-related eating disorder (SRED) is a parasomnia characterized by recurrent episodes of involuntary binge eating and drinking during the sleep period. The affected individuals often eat peculiar and at times bizarre combinations of high caloric food. Due to diminished or absent consciousness during these episodes, patients may ingest toxic substances or suffer injuries during food preparation (e.g. cutting oneself, burning oneself with very hot food or liquids) [2, 3]. In addition, they may not remember these events the next morning $[2,4]$. Finally, SRED may be associated with morning anorexia, sleep disruption, excessive weight gain and other adverse effects from excessive food intake, such as metabolic and dental problems.

There is some controversy as to whether SRED and NES are similar disorders situated at opposite ends of a common spectrum of pathophysiology, with different levels of consciousness, or whether they are distinct disorders with separate underlying pathophysiologies [1]. Although this diagnostic issue remains unresolved, the International Classification of Sleep Disorders, 2nd Edition, by not including a level of consciousness criterion for the parasomnia SRED, has not definitively distinguished between these two disorders, thus encouraging further research [1].

Herein we describe 2 patients with Parkinson's disease (PD) with typical symptoms of SRED. They belong to a cohort of $45 \mathrm{PD}$ patients that was prospectively followed at a tertiary outpatient clinic for sleep disorders. The clinical evaluation of both patients was performed by movement disorders and sleep specialists, a psychiatrist and a neuropsychologist. We performed an audio-video polysomnography (PSG) which included extended electroen- cephalogram montages (full-scalp electroencephalogram, positioning of leads according to the International 10-20 System), electromyography of the chin, anterior tibialis and brachioradialis muscles, electrocardiography, and measures of nasal and oral airflow, thoracic and abdominal respiratory effort and oxygen saturation. This study has been approved by the local ethics committee and has therefore been performed in accordance with the Declaration of Helsinki.

\section{Case Reports}

Case 1

A 28-year-old man with PD onset when 17 years old reported episodes of binge eating during sleep. The patient could not remember these events, during which he usually ate peculiar combinations or forms of food, such as raw meat or coffee mixed with butter. In addition, he reported having anorexia the next morning. These episodes started two years ago on a nightly basis, usually in the first third of the night. Six months before the beginning of these events he started to use pramipexole, increasing the dose over time to $4.5 \mathrm{mg} / \mathrm{day}$, with satisfactory control of his PD motor symptoms $(\mathrm{UPDRS}=27$, Hoehn and

\section{KARGER}

Fax +41613061234 E-Mail karger@karger.ch www.karger.com
Dr. Alan Luiz Eckeli, MD

Department of Neuroscience and Behavior, Ribeirão Preto

School of Medicine, University of São Paulo

Av. Bandeirantes 3900, São Paulo 14048-900 (Brazil)

Tel. +55 163602 2927, E-Mail eckeli_alan@yahoo.com.br 
Yahr $=2$, Schwab and England $=100 \%$ ). After the onset of these symptoms, he gained approximately $7 \mathrm{~kg}$. He denied having other impulse control disorders such as excessive sexual drive, pathological gambling, or excessive daytime wakeful eating and any symptoms of restless legs syndrome (RLS). He denied any other personal or family history of parasomnia and eating disorder and was not taking any sedative drug. A cognitive evaluation revealed mild cognitive deficits without functional impairment (Mini-Mental State Examination score $=25$ ). The psychiatric assessment showed no anxiety, psychosis, substance abuse/dependence, mood disorders or specific eating disorder (bulimia and anorexia nervosa). The PSG disclosed reduced sleep efficiency (78.1\%), relative reduction of $\mathrm{N} 1$ (5.9\%) and REM sleep (5.2\%), with a robust increase of N3 stage (i.e. delta sleep) (40.5\%) (fig. 1). Additionally, there were several episodes of confusional arousals, characterized by brief episodes of sitting up and looking around during N3 sleep that were not associated with epileptic discharges, respiratory events or periodic limb movements. Thus, his clinical history and polysomnographic findings were compatible with a diagnosis of SRED.

Besides providing sleep hygiene education, we gradually reduced the pramipexole dosage to $1.5 \mathrm{mg} /$ day in parallel with the introduction of $150 \mathrm{mg}$ levodopa per day. The new therapeutic schema provided satisfactory control of the parkinsonian symptoms and the patient showed a progressive reduction and finally a complete resolution of SRED symptoms.

\section{Case 2}

A 37-year-old man with PD since he was 32 years old reported that he had been experiencing, over the last 3 years, almost daily episodes of nighttime awakenings followed by conscious binge eating, when he used to eat sweets, associated with morning anorexia. In addition, approximately every 3 months over the last year he presented episodes of unconscious eating during sleep, witnessed by his wife, when he ate bizarre combinations of food, such as a mixture of chocolate, white bread and ketchup. After the onset of these symptoms, he gained $35 \mathrm{~kg}$. His medical history included episodes of sleep terrors and sleepwalking during childhood, occurrences of dream enactment associated with violent behavior in the last 15 years and snoring, witnessed apneas and excessive daytime sleepiness (Epworth Sleepiness Scale $=15$, normal value $<10$ ) during the last 3 years. He denied having any symptoms of RLS or family history of parasomnia and eating disorder. The psychiatric assessment showed no anxiety, psychosis, substance abuse/dependence, mood or other impulse disorders or specific eating disorder. His cognitive evaluation, was normal. At the time of evaluation he was taking pramipexole $4.5 \mathrm{mg} / \mathrm{day}$, amantadine $300 \mathrm{mg} / \mathrm{day}$, selegiline $10 \mathrm{mg} /$ day and biperiden $6 \mathrm{mg} /$ day, with satisfactory control of his PD motor symptoms $(\mathrm{UPDRS}=28$, Hoehn and Yahr $=1.5$, Schwab and England $=80 \%$ ). He was not taking any sedative drugs. He had been taking pramipexole for 5 years, without any dosage change in the last 4 years. A PSG (fig. 1) evidenced a good sleep efficiency (88.5\%), increased N1 (24.1\%) and reduced REM sleep (4.4\%), increased arousal index $(48.1 / \mathrm{h})$, increased respiratory disturbances index $(54.9 / \mathrm{h})$, and presence of oxygen desaturation episodes during sleep $\left(\mathrm{SaO}_{2}=88 \%\right)$ associated with respiratory events. Furthermore, REM sleep without atonia was observed, which, when combined with the clinical history of episodic, violent dream enactment, was compatible with the diagnosis of REM sleep behavior disorder (RBD) [3]. In summary, based on these clinical and polysomnographic findings, this patient was diagnosed with obstructive sleep apnea syndrome (OSAS), SRED, NES, and RBD. Also, the presence of SRED and RBD in this patient documented a new variant of the 'Parasomnia Overlap Disorder' (POD) involving $\mathrm{RBD}$ and a non-REM sleep parasomnia [3,5]. After treatment of the OSAS with a continuous positive airway pressure device, in addition to sleep hygiene education, the excessive daytime sleepiness and the conscious and unconscious episodes of nighttime eating were abolished.

\section{Discussion}

Sleep disorders affect up to $88 \%$ of patients with PD, leading to a negative impact on their quality of life $[6,7]$. There are several reports of distinct sleep disorders presented in patients with $\mathrm{PD}$, including OSAS, RBD, RLS, among others [8, 9]. As far as we know, this is the first description of SRED in patients with PD.
It is important to note that SRED is different from binge eating disorder (BED), described as part of impulse control disorder (ICD) in PD patients [14-16]. In BED, patients usually eat larger amounts of normal, nonbizarre combinations of food, during short periods of time which occur throughout the day. They also have an uncontrollable consumption of food, but have full consciousness during eating [15, 17, 18]. Until now, the literature has not clearly discriminated BED from SRED in PD patients. Additionally, previous reports of nocturnal eating in PD patients with drug-related compulsive eating may have included unrecognized cases of SRED $[16,19]$.

SRED has been associated with sleepwalking, RLS, periodic limb movement disorder, OSAS $[1,2,10]$ and with the use of various medications such as antidepressants, lithium, triazolam, olanzapine, risperidone, and zolpidem [2]. However, there are no reports of its occurrence after the initiation of pramipexole, as observed in case one. On the other hand, pramipexole has been implicated in several ICDs in PD patients $[11,12]$ and the association between SRED and drug-induced ICD has been theorized in a recent review about sleep disturbances in PD [13]. Even considering that in our first case we did not observe the existence of other symptoms related to ICD, such as hypersexuality, repetitive behavior or compulsive gambling, we decided to decrease the pramipexole daily dosage because there appeared to be a temporal, causal association between the SRED onset and the start of pramipexole therapy. Thus, cessation of SRED symptoms after the reduction of the pramipexole dosage suggests that pramipexole was the trigger for the onset of the SRED. We cannot exclude the possibility that the introduction of levodopa or the application of measures of sleep hygiene could have contributed to the amelioration of SRED episodes. Also, this case might represent a novel example of pramipexole-induced, isolated sleep-related ICD, without any daytime wakeful correlate. However, additional studies are necessary to evaluate the role of pramipexole in the development of SRED in PD patients.

Interestingly, Provini et al. [20], in a double blind, placebo-controlled trial, observed that low doses of pramipexole improved some symptoms of SRED. However, this study did not enroll PD patients, and used small pramipexole doses (up to 


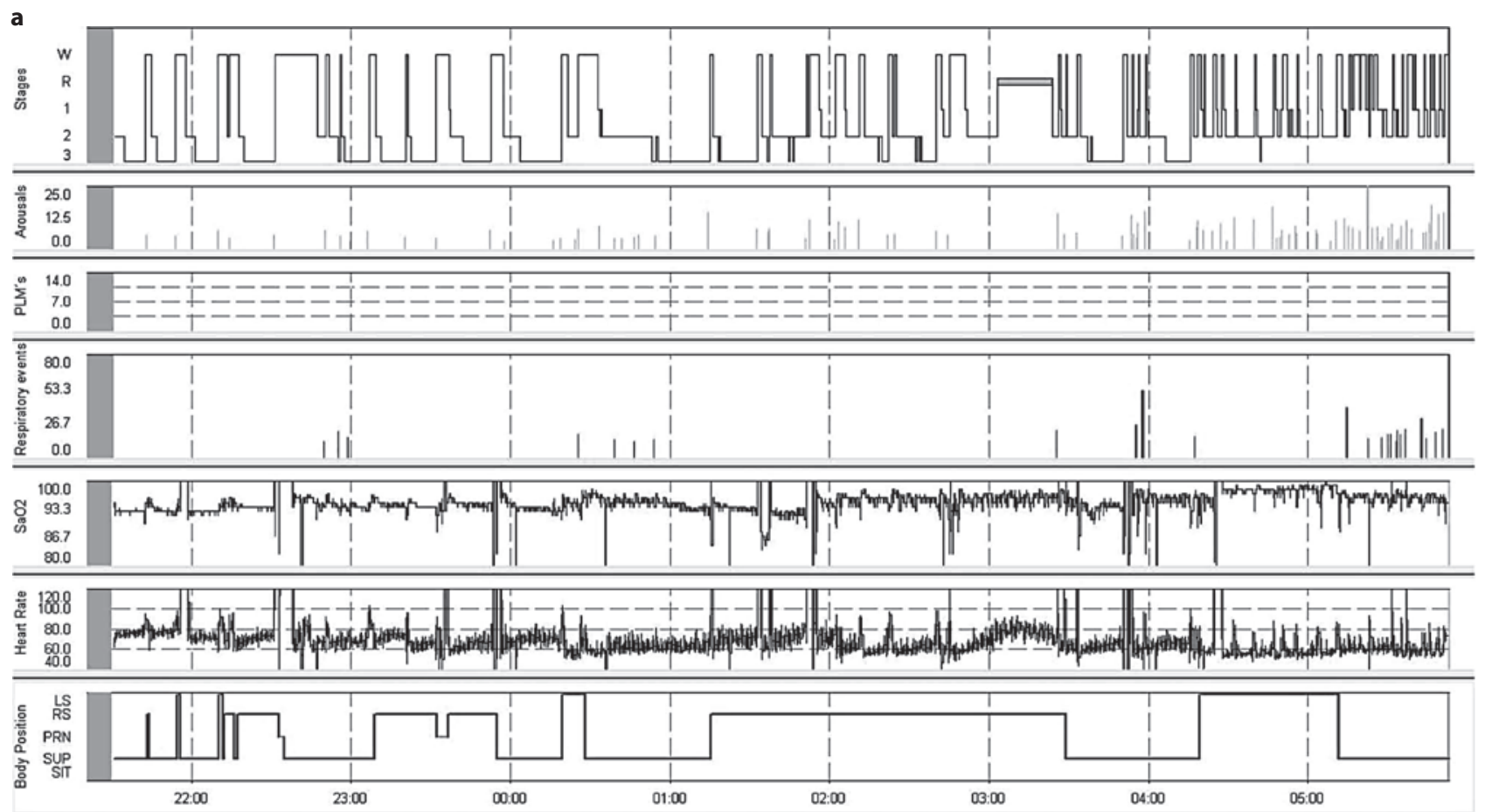

b

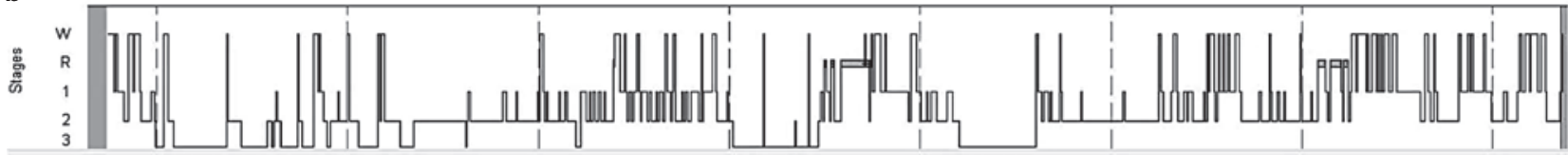

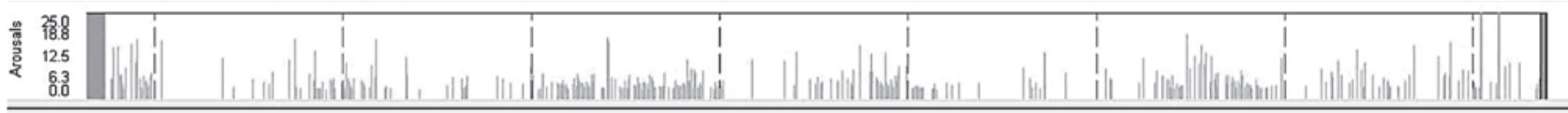

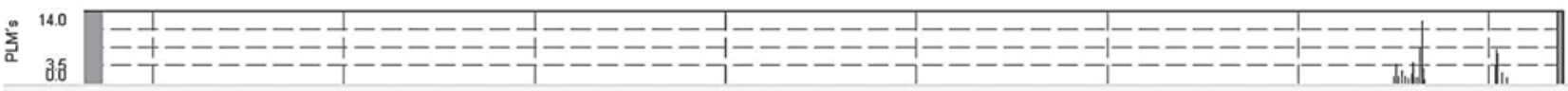

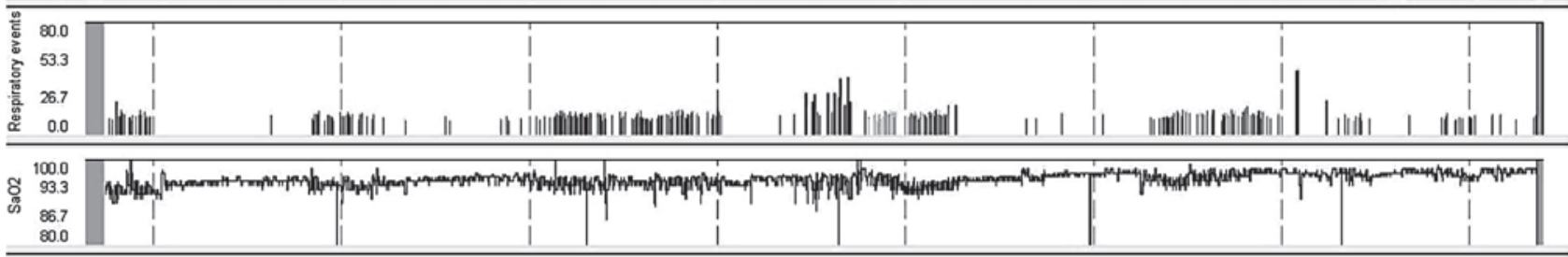

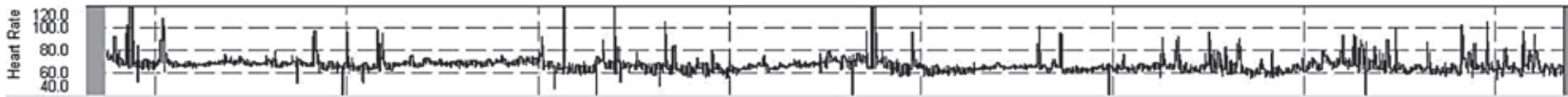

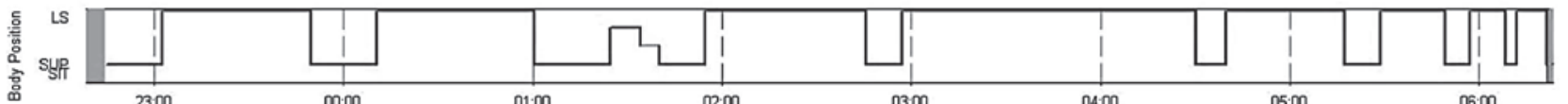

Fig. 1. a Case 1 hypnogram. At the onset of sleep stages, multiple awakenings during N3 sleep stage, corresponding to confusional awakenings, are observed. b Hypnogram of case 2. An excessive fragmentation of sleep in consequence of obstructive respiratory events (apneas and hypopneas) is observed. 
$0.36 \mathrm{mg} /$ day) during a short (2 weeks) treatment period. Besides, a case control study comparing RLS and nocturnal eating showed that patients who received dopaminergic therapy for RLS presented a tendency to have a higher risk of developing SRED $[10,19]$.

In case 2, we observed the coexistence of SRED with NES, OSAS and RBD. There have been reports of coexistence of SRED with OSAS, and some authors suggest that there is an improvement of SRED episodes after control of OSAS $[1,2]$, as was observed in our patient. For this reason, we decided to keep his antiparkinsonian drugs unchanged. The occurrence of RBD is common in $\mathrm{PD}$ patients [3], and may be coincidental in this case. Nevertheless, this case appears to represent a novel form of POD, with the coexistence of SRED and RBD in the same patient. To our knowledge, all previously reported cases of POD have involved sleepwalking, sleep terrors, and/or confusional arousals from slowwave sleep (and not SRED), together with $\mathrm{RBD}$. Also, this case appears to be another novel variant of POD (with SRED) associated with $\mathrm{PD}$, with 4 other cases being re- ported in a series of 6 PD cases with sleepwalking and RBD [21].

Since we had no previous experience or therapeutic guideline to follow, we decided to treat SRED according to the patient's clinical profile. Case 1 was successfully treated with the reduction of pramipexole daily dosage, while case 2 improved after treatment of abnormal sleep respiratory events.

It is also important to note the different epidemiological characteristics observed in these patients compared to groups of SRED patients previously reported [2]. In general, there is a female predominance, presence of positive family history and an increased index of periodic limb movements in PSG [2]. The two cases described above occurred in young men, there was no family history of PD and SRED, and both cases had a normal rate of periodic limb movements.

Concerning the characteristics of these patients, both had early-onset PD. It is well known that early-onset PD presents different genetic backgrounds and clinical profiles compared to late-onset patients [22]. In addition, early-onset PD patients have more chance to receive dopamine agonists, which might have a role in developing SRED, as discussed above. Beside this, younger age ( $\leq 65$ years) is associated with the occurrence of ICD and this age effect remained after controlling for dopamine agonist exposure [14]. Therefore, future studies will be necessary to evaluate whether SRED is more common in earlyonset PD patients.

In conclusion, it is important to draw attention to the pathological sleep behavior of SRED as another parasomnia being associated with PD (besides RBD and sleepwalking), and also presumably related to pramipexole intake, and the presence of OSAS, especially considering the risks of obesity and metabolic/dental comorbidities, and injuries that may occur during food seeking, preparation or ingestion.

\section{Disclosure Statement}

Potential conflict of interest: nothing to report. Full financial disclosure for the previous 12 months: nothing to report.

\section{References}

1 Winkelman JW: Sleep-related eating disorder and night eating syndrome: sleep disorders, eating disorders, or both? Sleep 2006; 29:876-877.

2 Howell MJ, Schenck CH, Crow SJ: A review of nighttime eating disorders. Sleep Med Rev 2009;13:23-34.

3 International Classification of Sleep Disorders, ed 2. American Academy of Sleep Medicine, 2005.

-4 Vetrugno R, Manconi M, Ferini-Strambi L, Provini F, Plazzi G, Montagna P: Nocturnal eating: sleep-related eating disorder or night eating syndrome? A videopolysomnographic study. Sleep 2006;29:949-954.

5 Schenck CH, Boyd JL, Mahowald MW: A parasomnia overlap disorder involving sleepwalking, sleep terrors, and REM sleep behavior disorder in 33 polysomnographically confirmed cases. Sleep 1997;20:972-981.

-6 Menza M, Dobkin RD, Marin H, Bienfait K: Sleep disturbances in Parkinson's disease. Mov Disord 2010; 25(suppl 1):S117-S122.

-7 Friedman JH, Millman RP: Sleep disturbances and Parkinson's disease. CNS Spectr 2008;13(suppl 4):12-17.

$\checkmark 8$ Comella CL: Sleep disorders in Parkinson's disease: an overview. Mov Disord 2007; 22(suppl 17):S367-S373.
-9 Iranzo A, Santamaria J, Tolosa E: The clinical and pathophysiological relevance of REM sleep behavior disorder in neurodegenerative diseases. Sleep Med Rev 2009; 13: 385-401.

10 Provini F, Antelmi E, Vignatelli L, et al: Association of restless legs syndrome with nocturnal eating: a case-control study. Mov Disord 2009;24:871-877.

$\checkmark 11$ Evans AH, Strafella AP, Weintraub D, Stacy M: Impulsive and compulsive behaviors in Parkinson's disease. Mov Disord 2009;24: 1561-1570.

12 Ceravolo R, Frosini D, Rossi C, Bonuccelli U: Impulse control disorders in Parkinson's disease: definition, epidemiology, risk factors, neurobiology and management. Parkinsonism Relat Disord 2009;15(suppl 4):S111-S115.

13 Chaudhuri KR, Logishetty K: Dopamine receptor agonists and sleep disturbances in Parkinson's disease. Parkinsonism Relat Disord 2009;15(suppl 4):S101-S104.

14 Weintraub D, Koester J, Potenza MN, et al: Impulse control disorders in Parkinson disease: a cross-sectional study of 3090 patients. Arch Neurol 2010;67:589-595.

15 Diagnostic and Statistical Manual of Mental Disorders, ed 4: American Psychiatric Association, 2000.
16 Nirenberg MJ, Waters C: Compulsive eating and weight gain related to dopamine agonist use. Mov Disord 2006;21:524-529.

$\checkmark 17$ Allison KC, Grilo CM, Masheb RM, Stunkard AJ: Binge eating disorder and night eating syndrome: a comparative study of disordered eating. J Consult Clin Psychol 2005; 73:1107-1115

18 Stunkard AJ, Allison KC, Geliebter A, Lundgren JD, Gluck ME, O’Reardon JP: Development of criteria for a diagnosis: lessons from the night eating syndrome. Compr Psychiatry 2009;50:391-399.

19 Nirenberg MJ, Waters C: Nocturnal eating in restless legs syndrome. Mov Disord 2010;25: 126-127.

20 Provini F, Albani F, Vetrugno R, Vignatelli L, Lombardi C, Plazzi G, et al: A pilot doubleblind placebo-controlled trial of low-dose pramipexole in sleep-related eating disorder. Eur J Neurol 2005;12:432-436.

21 Poryazova R, Waldvogel D, Bassetti CL: Sleepwalking in patients with Parkinson disease. Arch Neurol 2007;64:1524-1527.

22 Schrag A, Ben-Shlomo Y, Brown R, Marsden CD, Quinn N: Young-onset Parkinson's disease revisited - clinical features, natural history, and mortality. Mov Disord 1998;13: 885-894. 